\title{
Oscillations of the Eddington capture sphere
}

\author{
M. Wielgus ${ }^{1,2}$, A. Stahl ${ }^{3}$, M. Abramowicz ${ }^{3,1,4}$, and W. Kluźniak ${ }^{1}$ \\ ${ }^{1}$ Copernicus Astronomical Center, ul. Bartycka 18, 00-716 Warszawa, Poland \\ e-mail: wlodek@camk.edu.pl \\ 2 Institute of Micromechanics and Photonics, ul. św A. Boboli 8, 02-525 Warszawa, Poland \\ e-mail: maciek.wielgus@gmail.com \\ 3 Physics Department, Gothenburg University, 41296 Göteborg, Sweden \\ e-mail: gusstaad@student.gu.se; marek.abramowicz@physics.gu.se \\ ${ }^{4}$ Institute of Physics, Faculty of Philosophy and Science, Silesian University in Opava, Bezručovo nám. 13, 74601 Opava, \\ Czech Republic
}

Received 14 August 2012 / Accepted 28 August 2012

\section{ABSTRACT}

\begin{abstract}
We present a toy model of mildly super-Eddington, optically thin accretion onto a compact star in the Schwarzschild metric, which predicts periodic variations of luminosity when matter is supplied to the system at a constant accretion rate. These are related to the periodic appearance and disappearance of the Eddington capture sphere. In the model the frequency is found to vary inversely with the luminosity. If the input accretion rate varies (strictly) periodically, the luminosity variation is quasi-periodic, and the quality factor is inversely proportional to the relative amplitude of mass accretion fluctuations, with its largest value $Q \approx 1 /(10|\delta \dot{M} / \dot{M}|)$ attained in oscillations at about 1 to $2 \mathrm{kHz}$ frequencies for a $2 M_{\odot}$ star.
\end{abstract}

Key words. accretion, accretion disks - gravitation - relativistic processes - stars: neutron - X-rays: binaries

\section{Introduction}

Abramowicz et al. (1990, hereafter AEL) argued that the luminosity of a relativistic star that is accreting at a super-Eddington rate, should periodically change. They have shown that in the combined gravitational and radiation fields of a spherical, compact star, radially moving test particles are captured by a sphere on which the gravitational and radiative forces balance. This is because in Einstein's general relativity the radiative force diminishes more strongly with the distance than the gravitational force, and radiation may be super-Eddington close to the star, but sub-Eddington further away, reaching the Eddington value at the Eddington capture sphere (ECS), whose radius is given by a simple expression in the Schwarzschild coordinates (Phinney 1987),

$$
r_{\mathrm{ECS}}=\frac{2 R_{\mathrm{G}}}{1-\left(1-\frac{2 R_{\mathrm{G}}}{R^{2}}\right)^{2}\left(\frac{L}{L_{\mathrm{Edd}}}\right)^{2}} .
$$

Here $R$ is the radius of the star, $R_{\mathrm{G}}=G M / c^{2}$ its gravitational radius, and $L$, which is assumed to satisfy Eq. (2), is the stellar luminosity at its surface. Bini et al. (2009); Oh et al. (2011), and Stahl et al. (2012) have shown that the ECS captures particles from a wide class of non-radial orbits as well ${ }^{1}$, so the following discussion is not restricted to the case of radial accretion. However, it is important to keep in mind that the balance of forces necessary for the existence of the ECS requires a large, radial radiative flux. In non-spherical accretion this can only be realized in the optically thin regime.

In this paper we present a simple model in which the idea of periodic luminosity changes is realized. AEL assumed that

\footnotetext{
1 As shown in detail by $\mathrm{Oh}$ et al. (2011), azimuthal radiation drag efficiently removes particle's angular momentum, typically making motions in the combined gravitational and radiation fields asymptotically radial.
}

the radiation power was provided by the kinetic energy of the accreted particles, released when they hit the surface of the star. As stressed by Stahl et al. (2012), particles settle on the ECS rather gently, so the ECS itself is not particularly luminous. If all particles are captured at the Eddington sphere, they do not reach the surface of the star, and the stellar accretion luminosity goes to zero. When it does, and actually already when $L / L_{\text {Edd }}<(1-$ $\left.2 R_{\mathrm{G}} / R\right)^{-1 / 2}$, the ECS disappears, accretion is resumed by the star and eventually the luminosity may build up to its former value, so the ECS will reappear and accretion will stop again. Thus, the accretion process may be quasiperiodic, alternating between states of high luminosity and no luminosity.

\section{The model}

The toy model considered here assumes a steady ${ }^{2}$ supply of optically thin fluid at some distance above the stellar surface. As shown by Stahl et al. (2012), unless its velocity is extraordinarily large the fluid will settle on the ECS, regardless of its point of origin, if only

$\left(1-2 R_{\mathrm{G}} / R\right)^{-1 / 2}<L / L_{\mathrm{Edd}}<\left(1-2 R_{\mathrm{G}} / R\right)^{-1}$.

In our model the stellar luminosity will be assumed to be either zero, or to have a certain definite value, $L=L_{0}$, satisfying Eq. (2). In this sense the model states are binary (on-off). This property implies that the ECS is located at a specific radius, $r_{\mathrm{ECS}}=r_{0}>R$, whenever present (Eq. (1)). The model is described by three equations:

$$
\begin{aligned}
L_{\mathrm{ECS}}(t) & =\mu L_{*}\left(t-\delta t_{1}\right), \\
L_{*}(t) & =\eta \dot{M}_{*}\left(t-\delta t_{\mathrm{r}}\right), \\
\dot{M}_{*}(t) & = \begin{cases}\dot{M}_{0} & \text { if } L_{\mathrm{ECS}}\left(t-\delta t_{\mathrm{i}}\right)<\mu L_{0}, \\
0 & \text { if } L_{\mathrm{ECS}}\left(t-\delta t_{\mathrm{i}}\right) \geq \mu L_{0},\end{cases}
\end{aligned}
$$

2 The assumption of a constant $\dot{M}$ will be relaxed in Sect. 4 . 
where $L_{\mathrm{ECS}}$ is the stellar luminosity at the ECS, $L_{*}$ is the luminosity at the stellar surface, $\dot{M}_{*}$ is the accretion rate at the stellar surface, $\mu$ is a redshift factor between $R$ and $r_{0}, \eta$ is the conversion factor between accretion rate and stellar luminosity.

The simplest possibility, which we will ignore, is that $\eta \dot{M}_{0}<$ $L_{\mathrm{Edd}}\left(1-2 R_{\mathrm{G}} / R\right)^{-1 / 2}$, so the critical luminosity for establishing the ECS is never reached, and the equations describe a steady state solution. We will assume instead that $L_{0}=\eta \dot{M}_{0}$, i.e., the stellar luminosity in the "on" state has the necessary value to establish an ECS at the radius $r_{0}$. This assumption allows nontrivial time behaviour of the model system.

There are three timescales that determine the behaviour of the model, the reaction time $\delta t_{\mathrm{r}}$, which is the timescale for converting accreting matter into radiation, the light travel time $\delta t_{1}$, which is the travel time of light from the stellar surface to the $\mathrm{ECS}$, and the infall time $\delta t_{\mathrm{i}}$, which is the infall time of matter from the ECS to the stellar surface in the absence of radiation. Now, $\delta t_{1}$ and $\delta t_{\mathrm{i}}$ can be determined from $R_{\mathrm{G}}, R$ and $r_{0}$. So, for a given star these two timescales are a function of the stellar luminosity alone, i.e., of $\eta \dot{M}_{0}$. The reaction time introduces a phase shift between accretion and radiation at the stellar surface. In numerical examples we will adopt two extreme values $\delta t_{\mathrm{r}}=0$, or $\delta t_{\mathrm{r}}=0.1 \mathrm{~ms}$, the latter being the estimated cooling time of a clump of fluid that falls on a neutron star surface (Kluźniak et al. 1990).

In reality, the time delays would not be as sharp as we have assumed them to be. For instance, even at time $\delta t_{1}$ after the luminosity is turned off, particles outside the ECS suffer from radiation drag for an additional interval of time $\left(r-r_{0}\right) / c$, where $r$ is the position of the particle at time $\delta t_{1}+\left(r-r_{0}\right) / c$ after the radiation is turned off. Conversely, after the luminosity is turned on again, all particles between the ECS and the stellar surface will feel the full impact of radiation pressure after a delay of only $(r-R) / c<\delta t_{1}$, where again, $r$ is the position of the particle when the radiation front passes it, $(r-R) / c$ after the radiation is turned on. In the toy model, we neglect such effects entirely.

Combining Eqs. (3)-(5) we find that $\dot{M}_{*}(t)$ is determined by $\dot{M}_{*}(t-T)$, with $T=\delta t_{\mathrm{i}}+\delta t_{\mathrm{r}}+\delta t_{1}$ :

$$
\begin{aligned}
\dot{M}_{*}(t) & =\dot{M}_{0} \Theta\left(\mu L_{0}-L_{\mathrm{ECS}}\left(t-\delta t_{\mathrm{i}}\right)\right) \\
& =\dot{M}_{0} \Theta\left(L_{0}-L_{*}\left(t-\delta t_{\mathrm{i}}-\delta t_{1}\right)\right) \\
& =\dot{M}_{0} \Theta\left(\dot{M}_{0}-\dot{M}_{*}\left(t-\delta t_{\mathrm{i}}-\delta t_{1}-\delta t_{\mathrm{r}}\right)\right) \\
& =\dot{M}_{0}-\dot{M}_{*}(t-T),
\end{aligned}
$$

where $\Theta$ is the Heaviside step function. Thus,

$$
\begin{aligned}
\left\{\dot{M}_{*}(t-T)=\dot{M}_{0}\right\} & \Rightarrow\left\{\dot{M}_{*}(t)=0\right\}, \\
\left\{\dot{M}_{*}(t-T)=0\right\} & \Rightarrow\left\{\dot{M}_{*}(t)=\dot{M}_{0}\right\},
\end{aligned}
$$

and clearly, the model system shows periodic behaviour with the period $2 T$ :

$\dot{M}_{*}(t)=\dot{M}_{*}(t-2 T)$.

\section{Results of the model}

A typical behaviour of the model is shown in Fig. 1 which shows a solution of Eqs. (3)-(5) with $\delta t_{1}: \delta t_{\mathrm{i}}: \delta t_{\mathrm{r}}=8: 17: 10$. The black solid line traces $\dot{M}_{*}$. In the figure, we identify the time $b-a=$ $e-d=\delta t_{\mathrm{r}}$ as the reaction time, $c-b=h-e=\delta t_{1}$ as the light travel time, and $d-c=j-h=\delta t_{\mathrm{i}}$ as the infall time. The luminosities at the stellar surface and at the ECS are shifted

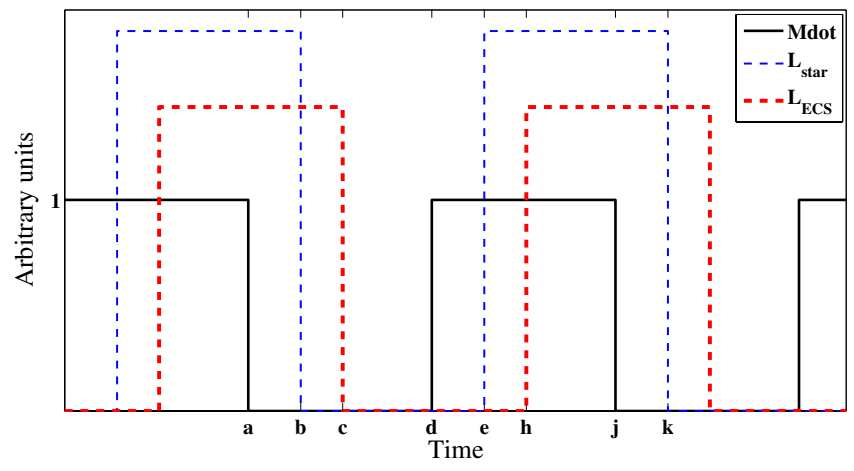

Fig. 1. Stellar accretion rate and luminosities at the stellar surface and at the ECS, according to the model.

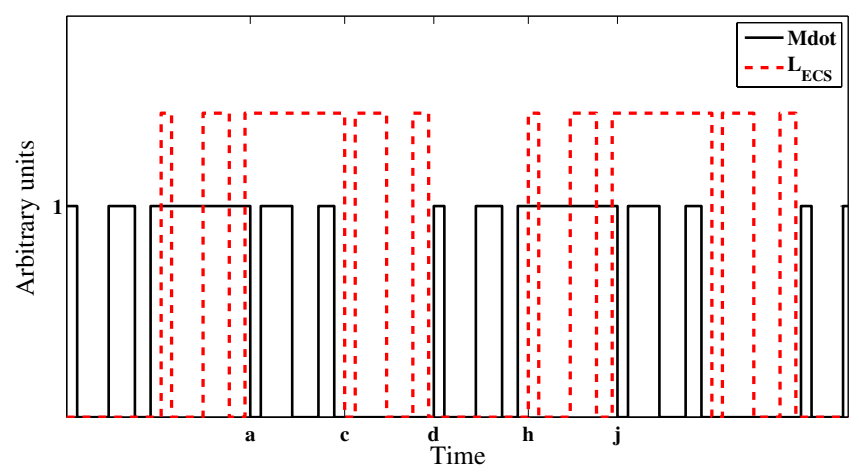

Fig. 2. Stellar accretion rate and the luminosity at the ECS, for another choice of initial conditions.

relative to the accretion rate by $\delta t_{\mathrm{r}}$ and $\delta t_{\mathrm{r}}+\delta t_{1}$, respectively. We note also that $d-a=j-d=h-c=k-e=e-b=\delta t_{\mathrm{r}}+\delta t_{1}+\delta t_{\mathrm{i}}=$ $T$, and the pulses are as long as the periods without activity. Thus the period of the oscillation is $P=2 T$, as expected from Eq. (6).

In constructing Fig. 1 it was assumed that matter first arrives at the stellar surface at $t=0$ and that there is a continual inflow of matter to the system at the rate $\dot{M}_{0}$ for all $t \geq 0$. Different initial conditions can lead to a more complicated pulse shape of $\dot{M}_{*}(t)$ for $t$ in the intervals $(2 n T, 2 n T+T)$, with its complement $\dot{M}_{*}(t)=\dot{M}_{0}-\dot{M}_{*}(t-T)$ in the intervals $(2 n T+T, 2 n T+2 T)$. Here, and elsewhere, $n=0,1,2,3 \ldots$ The state of accretion $\left(\dot{M}_{0}\right.$ or 0$)$ at time $t$ has no influence on the future value of $\dot{M}_{*}$ until the time $t+T$, so one can assume that at any instant in the initial interval $t \in[0, T)$ the accretion rate has any of the two values, 0 or $\dot{M}_{0}$, i.e., in this interval $\dot{M}_{*}(t)=\dot{M}_{0} g(t)$, where $g(t)$ is an arbitrary binary function, mapping the initial time interval into on-off states, $g:[0, T) \rightarrow\{0,1\}$. Figure 2 provides an example of such behaviour. Thus, in principle, the harmonic content of the signal may be quite rich, although the fundamental is still at $f=1 / P=1 /(2 T)$.

As can be seen from Eqs. (3)-(5) the model takes no account of accumulation of matter on the ECS, that is to say, here we ignore the arrival at $t=2(n+1) T$ (and at other moments as well in the case of Fig. 2), of the additional matter that had accumulated on the ECS. In reality, this matter would produce a brief flash of radiation of energy $\eta_{\mathrm{ECS}} \dot{M}_{0} \Delta T$, where $\eta_{\mathrm{ECS}}$ is the conversion efficiency to radiation of the kinetic energy of matter falling from the ECS, and $\Delta T$ is the accumulation time $(\Delta T=T$ in Fig. 1). If in the steady accretion phase matter is falling in from $r \gg r_{0}$, one would expect $\eta_{\text {ECS }} \ll \eta$.

In a physically realistic situation the infall time is the dominant time scale, $\delta t_{\mathrm{i}} \gg \delta t_{1}$. The radius of the ECS, and hence also 


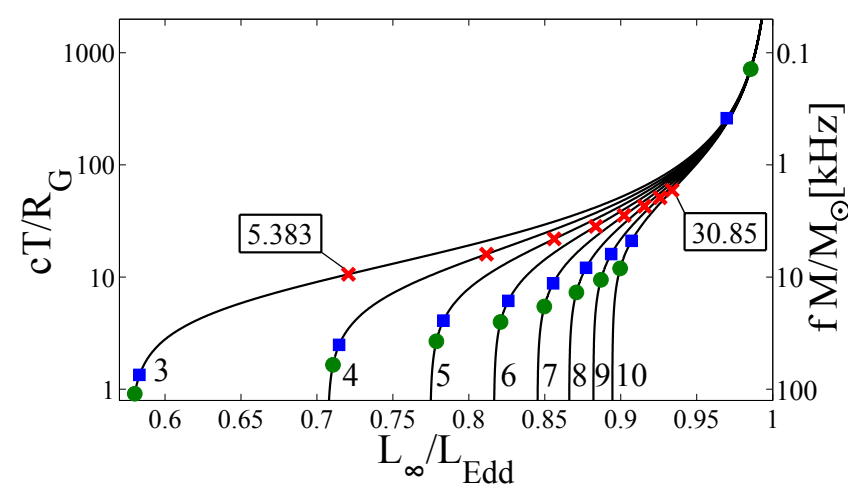

Fig. 3. The semi-period $T$ of the oscillation in geometrical units as a function of peak luminosity at infinity in Eddington units, when kinetic energy is instantaneously converted to luminosity $\left(\delta t_{\mathrm{r}}=0\right)$. The corresponding frequency $f=1 /(2 T)$ can be read off in $\mathrm{kHz}$ from the right vertical axis. The curves are labeled with the stellar radius $(3, \ldots, 10)$ in units of $R_{\mathrm{G}}$. Filled squares indicate a value of the slope $\operatorname{d} \log T / \operatorname{dlog} L_{\infty}=50$, filled circles a value of 100 , and the crosses the minimum value of the logarithmic derivative for each curve.

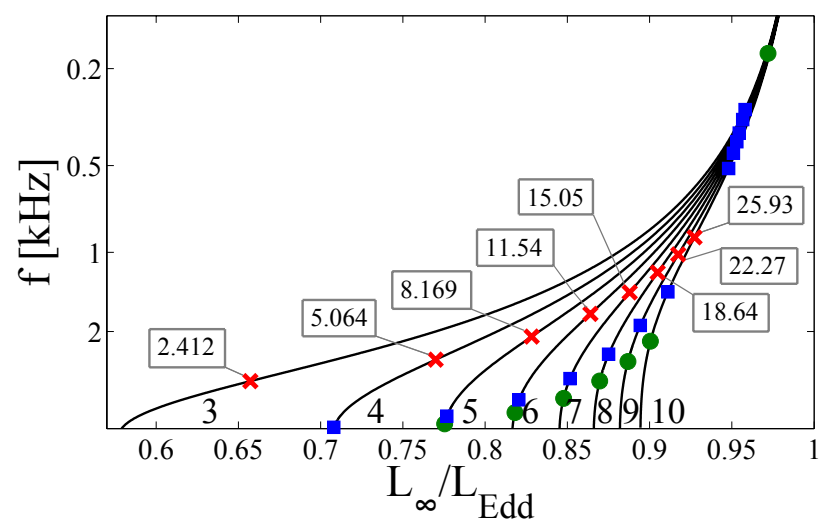

Fig. 4. The frequency of the oscillations as a function of peak luminosity at infinity in Eddington units, when there is a delay in converting kinetic energy to luminosity $\delta t_{\mathrm{r}}=0.1 \mathrm{~ms}$ (see Kluźniak et al. 1990). The stellar mass is assumed to be $M=2 M_{\odot}$. The curves are labeled with the stellar radius $(3, \ldots, 10)$ in units of $R_{\mathrm{G}}$. Filled squares indicate a value of the slope $\operatorname{dlog} T / \operatorname{dog} L_{\infty}=30$, filled circles a value of 50 , and the crosses the minimum value of the slope for each curve (boxed values).

the infall time, increases rapidly with $L_{\infty}$, and without bounds as $L_{\infty} \rightarrow L_{\mathrm{Edd}}$ :

$\frac{r_{\mathrm{ECS}}}{2 R_{\mathrm{G}}}=\left[1-\left(\frac{L_{\infty}}{L_{\mathrm{Edd}}}\right)^{2}\right]^{-1}$

Here, $L_{\infty}$ is the redshifted luminosity at infinity. Hence, except for the lowest values of the luminosity parameter $L_{0}$, the infall time $\delta t_{\mathrm{i}}$ is the dominant time scale, and the frequency of oscillations $f=1 /(2 T)$ varies inversely with the luminosity. Neglecting $\delta t_{\mathrm{r}}$, we can compute the semi-period $T$, and the frequency of oscillation as a function of the stellar radius $R$ and of the luminosity $L_{\infty}$ alone- $T$ and $f$ will, respectively, scale directly or inversely with $M$. These are shown in Fig. 3 for various stellar radii. Figure 4 shows the frequency as a function of luminosity, for a star with $M=2 M_{\odot}$, when $\delta t_{\mathrm{r}}=0.1 \mathrm{~ms}$.

\section{Conclusions and discussion}

We have shown that supplying mass to the vicinity of a compact star, continuously and at a constant accretion rate, can lead to periodic top-hat variations of luminosity, if only the mass accretion rate corresponds to a mildly super-Eddington luminosity at the stellar surface. This oscillatory behaviour of luminosity is related to the phenomenon of the ECS, which is a consequence of the interplay of radiation drag and general relativity.

If such an oscillation occurs in the real world, for example in the $\mathrm{Z}$ sources, where rapid variations of the inferred inner radius of the accretion disk have been reported (Lin et al. 2009), the actual mechanism is likely to be more complex than the strictly periodic oscillation in the toy model considered here. E.g., the accretion rate is not likely to be constant (contrary to our assumption of simple on-off behaviour at the stellar surface).

As a minor extension of the model, consider an accretion rate that is varying on a timescale comparable to the period of the ECS oscillator. Even strictly periodic variation of $\dot{M}_{0}$ will lead to a decoherence of the ECS oscillation, because the position $r_{0}$ of the ECS and (hence) the infall time from the ECS, and (hence) the oscillator frequency, are strongly varying functions of the luminosity, $L_{0}=\eta M_{0}$. Indeed,

$\frac{|\delta f|}{f}=\frac{|\delta T|}{T}=\frac{\mathrm{d} \ln T}{\mathrm{~d} \ln L_{\infty}} \frac{\left|\delta L_{\infty}\right|}{L_{\infty}}=\frac{\mathrm{d} \ln T}{\mathrm{~d} \ln L_{\infty}}\left|\frac{\delta \dot{M}_{0}}{\dot{M}_{0}}\right|$.

Thus, the $Q$ factor of the oscillation is

$Q=\frac{f}{|\delta f|}=\left|\frac{\dot{M}_{0}}{\delta \dot{M}_{0}}\right|\left(\frac{\mathrm{d} \ln T}{\mathrm{~d} \ln L_{\infty}}\right)^{-1}$.

We have indicated some values of the logarithmic derivative $\mathrm{d} \ln T / \mathrm{d} \ln L_{\infty}$ in Figs. 3, 4. Comparing the figures, we see that delayed emission (reaction time $\delta t_{\mathrm{r}}>0$ ) at the stellar surface acts as a low-pass filter, cutting out the highest frequencies. At the same time it improves the quality factor of the oscillator.

Accreting neutrons stars in low mass X-ray binaries are expected to have a radius in the range of 4 to $7 G M / c^{2}$ (Arnett \& Bowers 1977; Kluźniak \& Wagoner 1985), and a mass of about two solar masses. It is seen from Fig. 4 that the minimum values of the logarithmic derivative attained for radii of $4,5,6,7 G M / \mathrm{c}^{2}$ are between 5 and 15 . Hence, the maximum expected value of the oscillator quality factor would be $Q \approx 1 /\left(10\left|\delta \dot{M}_{0} / \dot{M}_{0}\right|\right)$, occurring for frequencies between about 1 and $2 \mathrm{kHz}$, as can be seen from Fig. 4.

Acknowledgements. Research supported in part by Polish NCN grants UMO2011/01/B/ST9/05439 and N N203 511238.

\section{References}

Abramowicz, M.A., Ellis, G. F. R., \& Lanza, A. 1990, ApJ, 361, 470 Arnett, W. D., \& Bowers, R. L. 1977, ApJS, 33, 415

Bini, D., Jantzen, R. T., \& Stella, L. 2009, Class. Q. Grav., 26, 055009

Kluźniak, W., \& Wagoner, R. V. 1985, ApJ, 297, 548

Kluźniak, W., Michelson, P., \& Wagoner, R. V. 1990, ApJ, 358, 538

Lin, D., Remillard, R. A., \& Homan, J. 2009, ApJ, 696, 1257

Oh, J. S., Hongsu, J., \& Hyung, M. L. 2011, New Astron., 16, 183

Phinney, E. S. 1987, in Superluminal Radio Sources, eds. J. A. Zensus, \& T. J.

Pearson (Cambridge: Cambridge University Press), 12

Stahl, A., Wielgus, M., Abramowicz, M. A., Kluźniak W., \& Yu, W. 2012, A\&A, in press, DOI: 10.1051/0004-6361/201220187 